\title{
The effect of saccades on number processing
}

\author{
DaVid E. Irwin ANd LaURa E. Thomas \\ University of Illinois at Urbana-Champaign, Urbana, Illinois
}

\begin{abstract}
Recent research has shown that saccadic eye movements interfere with dorsal-stream tasks such as judgments of object orientation, but not with ventral-stream tasks such as object recognition. Because saccade programming and execution also rely on the dorsal stream, it has been hypothesized that cognitive saccadic suppression occurs as a result of dual-task interference within the dorsal stream. Judging whether one number is larger or smaller than another (magnitude comparison) is a dorsal-stream task that relies especially on the right parietal cortex. In contrast, judging whether a number is odd or even (parity judgment) does not involve the dorsal stream. In the present study, one group of subjects judged whether two-digit numbers were greater than or less than 65 , whereas another group judged whether two-digit numbers were odd or even. Subjects in both groups made these judgments while making no, short, or long saccades. Saccade distance had no effect on parity judgments, but reaction times to make magnitude comparison judgments increased with saccade distance when the eyes moved from right to left. Because the right parietal cortex is instrumental in generating leftward saccades, these results provide further evidence for the hypothesis that cognitive suppression during saccades occurs as a result of dual-task interference within the dorsal stream.
\end{abstract}

People inspect the world by means of rapid eye movements called saccades. Saccades are separated by fixations during which the eyes are relatively still. Saccades occur about 3 times each second, or approximately 172,800 times during the course of the average day. Saccade duration depends on saccade distance, with the average duration during reading and picture viewing being approximately 30-50 msec (Rayner, 1978, 1998). Thus, the eyes are in motion about $90-150$ min each day.

It is well known that visual sensitivity is reduced during saccades, with the result that people do not ordinarily notice visual information sweeping across their retinas during saccades (Matin, 1974; Volkmann, 1986; Zuber $\&$ Stark, 1966). Not all visual information is suppressed during saccades, however; rather, suppression appears to be restricted to the magnocellular visual pathway, which carries information about luminance, contrast, and motion, while the parvocellular visual pathway (which carries information about color and form) remains largely unaffected (Burr, Morrone, \& Ross, 1994).

Perhaps surprisingly, recent evidence has shown that some cognitive processes are also suppressed during saccades (see Irwin, 2003, for a review); these include stimulus encoding (Sanders \& Houtmans, 1985), the resolution of degraded stimuli (Sanders \& Rath, 1991), memory scanning (van Duren, 1993), counting (Matin, Shao, \& Boff, 1993), mental rotation (Irwin \& Brockmole, 2000; Irwin \& Carlson-Radvansky, 1996), and changes in attentional scale from local to global levels of a form (Brockmole, Carlson, \& Irwin, 2002). Other cognitive processes are not suppressed during saccades, however, and these include response selection (van Duren \& Sanders, 1995), semantic priming (Henderson, 1992; Henderson, Pollatsek, \& Rayner, 1987), identity priming (Irwin, Carlson-Radvansky, \& Andrews, 1995), and word recognition and word identification (Irwin, 1998).

The distinction between the processes that are suppressed during saccades and those that are not corresponds reasonably well to the distinction between dorsal and ventral processing streams in vision. To review, Mishkin, Ungerleider, and Macko (1983) proposed that two functionally separable streams of visual processing exist in the brain. The ventral stream projects to areas of inferotemporal cortex and has been hypothesized to be responsible for perceptual recognition and cognitive representations of objects, whereas the dorsal stream projects to the posterior parietal cortex and has been hypothesized to be responsible for visuospatial transformations and for representing an object's location in space (but see Goodale \& Milner, 1992, and Merigan \& Maunsell, 1993, for alternative views). The studies of cognitive saccadic suppression summarized above suggest that dorsal stream tasks such as mental rotation and changes of attentional scale are suppressed during saccades, whereas ventral stream tasks such as priming and word recognition are not.

Because saccade generation and saccade execution also involve the dorsal stream, especially the parietal cortex (e.g., Schall, 1995), Irwin and Brockmole (2004) hypothesized that suppression of cognitive processing during saccades might occur as a result of dual-task interference within the dorsal stream. They examined this hypothesis in two experiments. In one, they found that saccades had

D. E. Irwin, irwin@uiuc.edu 
no effect on performance when subjects discriminated pictures of objects from pictures of nonobjects, but that saccades interfered with performance when subjects had to judge which direction an object was facing-left or right. That is, saccades did not interfere with object recognition, a prototypical ventral stream task, but they did interfere with judgments of object orientation, a prototypical dorsal stream task.

The purpose of the present research was to investigate further the hypothesis that saccades interfere with cognitive processes that rely on the dorsal stream. To do this, we investigated the effect of saccades on a magnitude comparison task extensively studied by Dehaene and his colleagues (e.g., Dehaene, Dupoux, \& Mehler, 1990; Pinel, Dehaene, Rivière, \& Le Bihan, 2001). In this task, on each trial subjects are presented with a two-digit number and they must respond whether the number is greater than or less than 65. Some of the numbers are close to 65 , whereas others are farther away. Dehaene and his colleagues have found that reaction time (RT) in this task is generally longer for numbers close to 65 than for numbers farther away, regardless of whether the numbers are presented in verbal or Arabic form. They have proposed that subjects inspect an internal spatial map or mental "number line" in order to perform this task, so that numbers that are close together require more time to discriminate. In support of this idea, in a number of neuroimaging and ERP studies, Dehaene and his colleagues have found that the magnitude comparison task activates the parietal cortex, especially the right parietal cortex (Chochon, Cohen, van de Moortele, \& Dehaene, 1999; Dehaene, 1996), and that the amount of parietal activation is also greater for numbers close to 65 than for numbers farther away, mirroring the RT data (Pinel et al., 2001). Because the parietal cortex is instrumental in the programming of saccadic eye movements, we hypothesized that saccades should interfere with magnitude comparison. Interference should be greatest for numbers close to 65 because parietal activation is greatest for these numbers, and interference might also depend on saccade direction, because the magnitude comparison task activates the right parietal cortex more than the left.

To ensure that any effects we observed were indeed due to magnitude comparison and not to other possible stimulus or response factors associated with numbers, we also collected data from a control condition in which subjects judged the parity of two-digit numbers (i.e., judgments of whether a number is odd or even). Unlike magnitude comparison, this judgment does not rely on spatial processing in the dorsal stream but rather on verbal processing and conceptual knowledge in the left frontal and temporal language areas (Dehaene \& Cohen, 1991; Dehaene, Piazza, Pinel, \& Cohen, 2003; Hubbard, Piazza, Pinel, \& Dehaene, $2005)$. These areas play no role in saccade programming or saccade execution, so we predicted that saccades would not interfere with performance in this task.

\section{METHOD}

\section{Participants}

Two groups of 16 students each participated in one experimental session approximately $60 \mathrm{~min}$ long. One group performed the mag- nitude comparison task; the other group performed the parity judgment task. All participants had normal or corrected-to-normal vision and were naive with respect to the experimental hypotheses.

\section{Apparatus}

A Dell Optiplex GX270 computer with a 21-in. SVGA color monitor was used to present the stimuli and to control the timing of stimulus onset and offset. The refresh rate was $85.07 \mathrm{~Hz}$ and the screen resolution was 800 pixels horizontally $\times 600$ pixels vertically. Viewing distance was held constant by a chinrest placed $49 \mathrm{~cm}$ from the monitor. At this viewing distance, the display monitor subtended $45^{\circ}$ of visual angle horizontally and $33.75^{\circ}$ vertically. Eye movements and participants' responses were recorded using an Eyelink II eyetracker (SR Research). The eye tracker computed the center and size of participants' pupils by utilizing infrared video-based technology and used an infrared head-tracking system to correct for minor changes in head position across trials. The eye tracker had $500-\mathrm{Hz}$ temporal resolution and $0.2^{\circ}$ spatial resolution. Participants completed a calibration procedure before each block of trials and a drift correction procedure at the beginning of each trial to compensate for minor changes in calibration across trials.

\section{Procedure}

The main (dual-task, number processing plus eye movement) procedure is shown in Figure 1. A subject began each trial by fixating a filled circle $\left(0.8^{\circ}\right.$ in diameter, which also served as the drift correction stimulus for the eyetracker) on one side of the display, $2.5^{\circ}$ from either the left or the right side of the monitor. The subject then pressed a button to initiate the trial sequence. This erased the fixation dot, and after a delay of $400 \mathrm{msec}$, a small open circle $\left(1^{\circ}\right.$ in diameter) was presented either $7^{\circ}$ or $40^{\circ}$ away. The subject was instructed to saccade to the open circle (saccade target) as soon as it appeared, but of course the eyes do not move instantaneously. After a delay of $94 \mathrm{msec}$, while the subject was still fixating the original fixation location, the stimulus number $\left(2^{\circ}\right.$ wide and $1.3^{\circ}$ high $)$ was presented for $71 \mathrm{msec}$ at that location. Approximately $125 \mathrm{msec}$ later (on average), the saccade was initiated to the saccade target. Thus, the eyes moved approximately $200 \mathrm{msec}$ after number onset, which corresponds to the time when magnitude comparison effects begin to emerge in the parietal cortex (Dehaene, 1996). Subjects in the magnitude comparison group were instructed to decide whether the stimulus was less than or greater than 65 while moving their eyes. These subjects pressed a trigger with the left hand if the stimulus was less than 65 and a trigger with the right hand if the stimulus was greater than 65 . Subjects in the parity judgment group were instructed to decide whether the stimulus was odd or even while

Fixation:

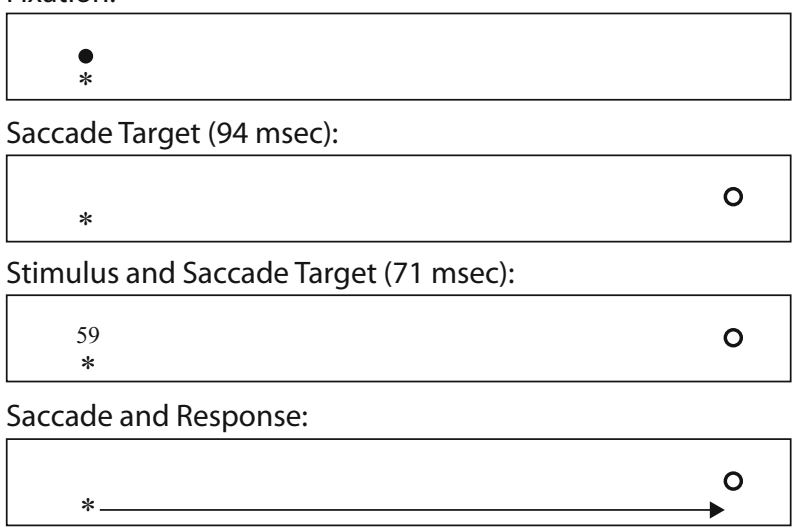

Figure 1. Sequence of events (from top to bottom) for number processing during an eye movement trial; the asterisk represents eye position. 
moving their eyes. These subjects pressed a trigger with the left hand if the stimulus was odd and a trigger with the right hand if the stimulus was even. Response time and accuracy were recorded from all subjects. Saccade distance $\left(7^{\circ}\right.$ vs. $\left.40^{\circ}\right)$ was sequenced randomly across trials, while saccade direction was blocked. Each subject completed four blocks of 58 trials each, with saccade direction (left vs. right) alternating across blocks. The first 10 trials in each block were practice; on these trials, the stimulus numbers were drawn from the sets 31-40 and 90-99. On experimental trials (i.e., the remaining 48 trials in each block), the stimulus numbers were drawn from the set 41-89, excluding 65. Across blocks, each subject judged each number in the experimental set for each saccade distance and for each saccade direction.

In addition to the dual-task procedure described above, each subject also completed two blocks of a single-task control condition in which their number-processing task was performed without eye movements; the format and timing of events on these trials was as described above, but subjects maintained fixation on the original fixation location throughout the trial. Each subject also completed two blocks of a single-task control condition in which eye movements were made without performing magnitude comparison. The procedure on these trials was identical to that of the dual-task procedure, except that the "stimulus" consisted of the letters XX instead of a two-digit number and no manual response was made to the stimulus. Each block consisted of 40 trials, 20 in each direction, with saccade distance sequenced randomly across trials.

The single- and dual-task procedures were completed in the following order: 58 number processing trials without saccades; 40 saccade-only trials; 20 practice trials requiring number processing during saccades (using the stimulus sets 31-40 and 90-99); four blocks of 58 trials each requiring number processing during saccades; 40 saccade-only trials; 58 number-processing trials without saccades.

\section{RESULTS}

The single-task control conditions provide baseline information about performance of one task (number processing or saccade execution) in the absence of the other. We consider those results before discussing the results of main interest, performance in the dual-task conditions in which number processing occurred during the saccade.

\section{Single-Task Performance: Number \\ Processing Without Saccades}

Magnitude comparison. The results of the no-saccade magnitude comparison condition replicated those of Dehaene and colleagues; it took subjects longer to decide whether a number was greater than or less than 65 when that number was close to 65 than when it was far away numerically. The RT data (correct trials only, measured from the onset of the stimulus number) were analyzed in a oneway ANOVA with three levels of numerical distance: near (stimulus numbers 60-69), medium (stimulus numbers 50-59 and 70-79), and far (stimulus numbers 40-49 and $80-89)$. Trials in which the eyes moved or blinks occurred were eliminated from analysis $(72.4 \%$ of the trials were accepted). The main effect of numerical distance (near $=$ $563 \mathrm{msec}$, medium $=490 \mathrm{msec}$, far $=460 \mathrm{msec}$ ) was significant $\left[F(2,30)=26.5, M S_{\mathrm{e}}=1,703.6, p<.001\right]$. The error term from this ANOVA was used to construct a $95 \%$ confidence interval for the difference between two means; this value was $\pm 26.6 \mathrm{msec}$, indicating that all pairwise comparisons were significant. Analysis of the accuracy data mirrored these results (near $=83.8 \%$, medium $=$ $93.1 \%$, far $=96.4 \%)\left[F(2,30)=6.8, M S_{\mathrm{e}}=0.01, p<\right.$ $.005]$, although the accuracy difference for medium versus far was not significant $(95 \%$ confidence interval for the difference between two means $= \pm 6.4 \%$ ).

Parity judgment. Trials in which the eyes moved or blinks occurred were eliminated from analysis $(72.9 \%$ of the trials were accepted). The mean RT to perform the parity judgment task (correct trials only, measured from the onset of the stimulus number) was $508 \mathrm{msec}$, quite similar to the mean RT in the magnitude comparison task (504 msec). The mean accuracy was $93.4 \%$, similar to the mean accuracy in the magnitude comparison task $(91.1 \%$, the difference being due to low accuracy for "near" numbers in the magnitude comparison task).

To evaluate whether the difficulty associated with processing numbers near 65 for the magnitude comparison group was indeed due to the demands of magnitude comparison, the mean RT data for the parity judgment subjects were also analyzed in a one-way ANOVA in terms of the same three numerical distance categories used for the magnitude comparison subjects (although of course distance from 65 is irrelevant to determining whether a number is odd or even). As expected, the main effect of numerical distance $($ near $=514 \mathrm{msec}$, medium $=507 \mathrm{msec}$, far $=507 \mathrm{msec}$ ) was not significant for the parity judgment subjects $\left[F(2,30)=0.2, M S_{\mathrm{e}}=1,076.6, p>.75\right]$. Numerical distance $($ near $=93.5 \%$, medium $=93.8 \%$, far $=92.9 \%$ ) also had no effect on accuracy in this task $\left[F(2,30)=0.2, M S_{\mathrm{e}}=0.002, p>.85\right]$.

In sum, in terms of overall performance (i.e., mean RT and mean accuracy), magnitude comparison and parity judgment appear to have been at least approximately equal in difficulty. Responding to numbers close to 65 was difficult only when magnitude comparison was involved, however. This implies that it is the process of magnitude comparison that hinders responding to numbers close to 65 , then, rather than anything inherently difficult about encoding or retrieving nonmagnitude information about these numbers.

\section{Single-Task Performance: Saccades Without Number Processing}

Magnitude comparison subjects. In the saccadeonly control condition, subjects executed long and short saccades in both directions without engaging in any number processing (as described above, the letters "XX" appeared in place of any stimulus number). Several aspects of saccade performance were measured and evaluated in ANOVAs with saccade direction and saccade distance as factors. Initial fixation location was affected by saccade direction $\left[F(1,15)=25.6, M S_{\mathrm{e}}=315.8, p<.001\right]$, but not by saccade distance $(F<1)$; subjects fixated $0.4^{\circ}$ to the right of the initial fixation location when the eyes moved to the right, but $0.8^{\circ}$ to the left of the initial fixation location when the eyes moved to the left (i.e., in both cases, fixation was biased in the direction of the upcoming saccade). Neither saccade direction nor saccade distance had any effect on saccade latency $(205 \mathrm{msec})$, however. Saccade direction had no effect on saccade distance $\left(6.3^{\circ}\right.$ 
on $7^{\circ}$-saccade trials and $35.6^{\circ}$ on $40^{\circ}$-saccade trials) or on average saccade velocity $\left(169.4^{\circ} / \mathrm{sec}\right.$ on $7^{\circ}$-saccade trials and $339.4^{\circ} / \mathrm{sec}$ on $40^{\circ}$-saccade trials). There was a significant interaction between saccade direction and saccade distance on saccade duration, however $[F(1,15)=5.4$, $\left.M S_{\mathrm{e}}=14.8, p<.05\right]$; saccade duration for $7^{\circ}$ saccades did not vary with saccade direction (rightward $=37.4 \mathrm{msec}$, leftward $=37.0 \mathrm{msec}$ ), but for unknown reasons, saccade duration for $40^{\circ}$ saccades was shorter when the eyes moved to the right $(104.1 \mathrm{msec})$ than when the eyes moved to the left (108.2 msec).

Parity judgment subjects. The results for the parity judgment subjects were quite similar to those for the magnitude comparison subjects (as they should have been, since subjects only moved their eyes in this part of the experiment). Initial fixation location was affected by saccade direction $\left[F(1,15)=35.5, M S_{\mathrm{e}}=2,904, p<\right.$ $.001]$, but not by saccade distance $(F<1)$; subjects fixated $1.3^{\circ}$ to the right of the initial fixation location when the eyes moved to the right, but $1.2^{\circ}$ to the left of the initial fixation location when the eyes moved to the left (i.e., in both cases fixation was biased in the direction of the upcoming saccade). Neither saccade direction nor saccade distance had any effect on saccade latency $(188 \mathrm{msec})$. Saccade direction had no effect on saccade distance $\left(5.8^{\circ}\right.$ on $7^{\circ}$-saccade trials and $29.8^{\circ}$ on $40^{\circ}$-saccade trials) or on average saccade velocity $\left(157.3^{\circ} / \mathrm{sec}\right.$ on $7^{\circ}$-saccade trials and $298.7^{\circ} / \mathrm{sec}$ on $40^{\circ}$-saccade trials). Only saccade distance affected saccade duration $\left(7^{\circ}\right.$ saccades $=36.6 \mathrm{msec}$, $40^{\circ}$ saccades $=99.7 \mathrm{msec}$ ).

\section{Dual-Task Performance}

The dual-task (number processing plus eye movement) trials allowed us to determine whether saccades and number processing interfere with each other. Trials were deleted from analysis if no saccade was made, if a blink occurred, or if the stimulus number was fixated for less than $25 \mathrm{msec}$ or for greater than $400 \mathrm{msec}$ before the eyes moved. On the basis of these criteria, $82.5 \%$ of the dualtask trials were accepted for analysis in the magnitude comparison group, whereas $78.6 \%$ were accepted in the parity judgment group. The percentage of accepted trials did not vary with saccade distance, saccade direction, or numerical distance, for either group of subjects. For each group, we will discuss first whether number processing affected saccades, and then whether saccades affected number processing.

\section{Effects of Number Processing on Saccades}

Saccades during magnitude comparison. Neither saccade direction nor saccade distance had any effect on initial fixation location (all $F \mathrm{~s}<1.3$ ); the eyes were fixated $0.3^{\circ}$ to the left of the initial fixation location in all conditions, in the direction of the first digit in the stimulus number. Saccade latency was affected by saccade distance only $\left[F(1,15)=15.0, M S_{\mathrm{e}}=2,656, p<.005\right]$; the latency for $7^{\circ}$ saccades was $297 \mathrm{msec}$, whereas the latency for $40^{\circ}$ saccades was $268 \mathrm{msec}$. Because the saccade target was presented $94 \mathrm{msec}$ before the stimulus number, this means that presaccadic processing time for the stimulus number was $203 \mathrm{msec}$ on $7^{\circ}$-saccade trials and $174 \mathrm{msec}$ on $40^{\circ}$-saccade trials (recall that the stimulus number itself was presented for only $71 \mathrm{msec}$, but the eyes lingered at its location for another 100-130 msec before moving to the saccade target location). Saccade direction had no effect on saccade distance $\left(6.7^{\circ}\right.$ on $7^{\circ}$-saccade trials and $35.6^{\circ}$ on $40^{\circ}$-saccade trials), but saccades to the left had a lower average velocity $\left(245.9^{\circ} / \mathrm{sec}\right)$ than did saccades to the right $\left(256.4^{\circ} / \mathrm{sec}\right)\left[F(1,15)=7.4, M S_{\mathrm{e}}=720, p<\right.$ $.02]$; as in the saccade-only control condition, average saccade velocity was lower for $7^{\circ}$ saccades $\left(171.5^{\circ} / \mathrm{sec}\right)$ than for $40^{\circ}$ saccades $\left(330.8^{\circ} / \mathrm{sec}\right)$. Only saccade distance affected saccade duration (for $7^{\circ}$ saccades, $38.9 \mathrm{msec}$; for $40^{\circ}$ saccades, $108.5 \mathrm{msec}$ ).

In sum, eye movement behavior during magnitude comparison differed from eye movement behavior in the absence of magnitude comparison in several ways; perhaps not surprisingly, fixation duration was short and the eyes drifted toward the saccade target when no number stimulus was present (i.e., in the saccade-only condition), but the eyes fixated the first digit in the stimulus number when it was present and subjects fixated it for a longer duration. Saccade distances and saccade durations differed little across single-task and dual-task conditions, but it is perhaps noteworthy that saccade direction affected average saccade velocity in the dual-task condition but not in the single-task condition; this result suggests that the cognitive task of magnitude comparison slowed saccades that were moving from right to left. This is discussed further below.

Saccades during parity judgments. Neither saccade direction nor saccade distance had any effect on initial fixation location (all $F \mathrm{~s}<1.8$ ); the eyes were fixated $0.3^{\circ}$ to the left of the initial fixation location in all conditions, in the direction of the first digit in the stimulus number. Saccade latency was affected by saccade distance only $\left[F(1,15)=9.3, M S_{\mathrm{e}}=1,688, p<.01\right]$; the latency for $7^{\circ}$ saccades was $287 \mathrm{msec}$, whereas the latency for $40^{\circ}$ saccades was $265 \mathrm{msec}$. Thus, the presaccadic processing time for the stimulus number was $193 \mathrm{msec}$ on $7^{\circ}$-saccade trials and $171 \mathrm{msec}$ on $40^{\circ}$-saccade trials. These values are very similar to those for the magnitude comparison group. Saccade direction had no effect on saccade distance $\left(6.0^{\circ}\right.$ on $7^{\circ}$-saccade trials and $31.8^{\circ}$ on $40^{\circ}$-saccade trials) or on average velocity $\left(227.1^{\circ} / \mathrm{sec}\right)$; as in the saccade-only control condition, average saccade velocity was lower for $7^{\circ}$ saccades $\left(154.5^{\circ} / \mathrm{sec}\right)$ than for $40^{\circ}$ saccades $\left(299.6^{\circ} / \mathrm{sec}\right)$. Only saccade distance affected saccade duration (for $7^{\circ}$ saccades, $38.7 \mathrm{msec}$; for $40^{\circ}$ saccades, $107.1 \mathrm{msec}$ ).

In sum, for the most part, parity judgment affected eye movement behavior in the same way that magnitude comparison did; in both cases, fixation duration was shorter and the eyes drifted toward the saccade target when no number stimulus was present, whereas the eyes fixated the first digit in the stimulus number when it was present and it was fixated for a longer duration. Saccade distances and saccade durations differed little across single-task and dual-task conditions. One potentially significant difference is that saccade direction affected average saccade velocity in the magnitude comparison task but not in the 
parity judgment task. This result suggests that whereas magnitude comparison slowed saccades that were moving from right to left, parity judgments did not.

\section{Effects of Saccades on Number Processing}

The primary question of interest in this study is whether saccades interfere with number processing; if they do, then number processing should be influenced by saccade distance. In particular, if number processing is suppressed during saccades, then RT should be longer in the $40^{\circ}$-saccade condition than in the $7^{\circ}$-saccade condition; if suppression is complete, the RT difference should be approximately $70 \mathrm{msec}$, the difference in duration between long and short saccades. In contrast, if number processing is not suppressed during saccades, then RT should be similar in the $7^{\circ}$ - and $40^{\circ}$-saccade conditions.

Magnitude comparison during saccades. As discussed in the introduction, the dual-task interference hypothesis predicts that saccades should interfere with magnitude comparison because magnitude comparison and saccade programming and execution all rely on neural processing in the parietal cortex. We also hypothesized that saccade direction might influence the results, because parietal activation during magnitude comparison is often larger in the right than in the left hemisphere. Figure 2 shows the mean RT in the magnitude comparison task as a function of saccade direction, saccade distance, and numerical distance (near, medium, and far, as defined earlier); the results of the no-saccade control condition are included for comparison. Inspection of Figure 2 shows that magnitude comparison RT was longer in the dual-task condition (i.e., magnitude comparison during saccades) than in the single-task control condition (magnitude comparison without saccades), but this does not necessarily mean that processing was suppressed during saccades. It might instead just reflect a general dual-task cost not specific to saccades, because on no-saccade trials subjects had to do only one thing - judge the number - whereas on saccade trials they had to do two things - judge the number plus move their eyes. To determine whether processing is suppressed during saccades per se, RT in the $7^{\circ}$-saccade condition must be compared with RT in the $40^{\circ}$-saccade condition. This was done in a three-way ANOVA with the factors of saccade direction (right vs. left), saccade distance $\left(7^{\circ}\right.$ vs. $\left.40^{\circ}\right)$, and numerical distance (near, medium, far). All main effects and interactions (except for saccade direction $\times$ numerical distance) were significant or marginally significant in this ANOVA, so the error term for the three-way interaction of saccade direction $\times$ saccade distance $\times$ numerical distance $\left[F(2,30)=3.2, M S_{\mathrm{e}}=\right.$ $1,472, p=.056]$ was used to construct a $95 \%$ confidence interval for the difference between two means to test the planned comparisons of interest (Winer, 1971, p. 384); this value was $\pm 24.7 \mathrm{msec}$. On the basis of this value, we can conclude that when the eyes moved to the right, saccade distance had no significant effect on RT at any numerical distance; when the eyes moved to the left, however, RT for numbers close to the standard (i.e., near trials) was significantly slower when subjects made $40^{\circ}$ saccades $(660 \mathrm{msec})$ than when they made $7^{\circ}$ saccades $(583 \mathrm{msec})$.
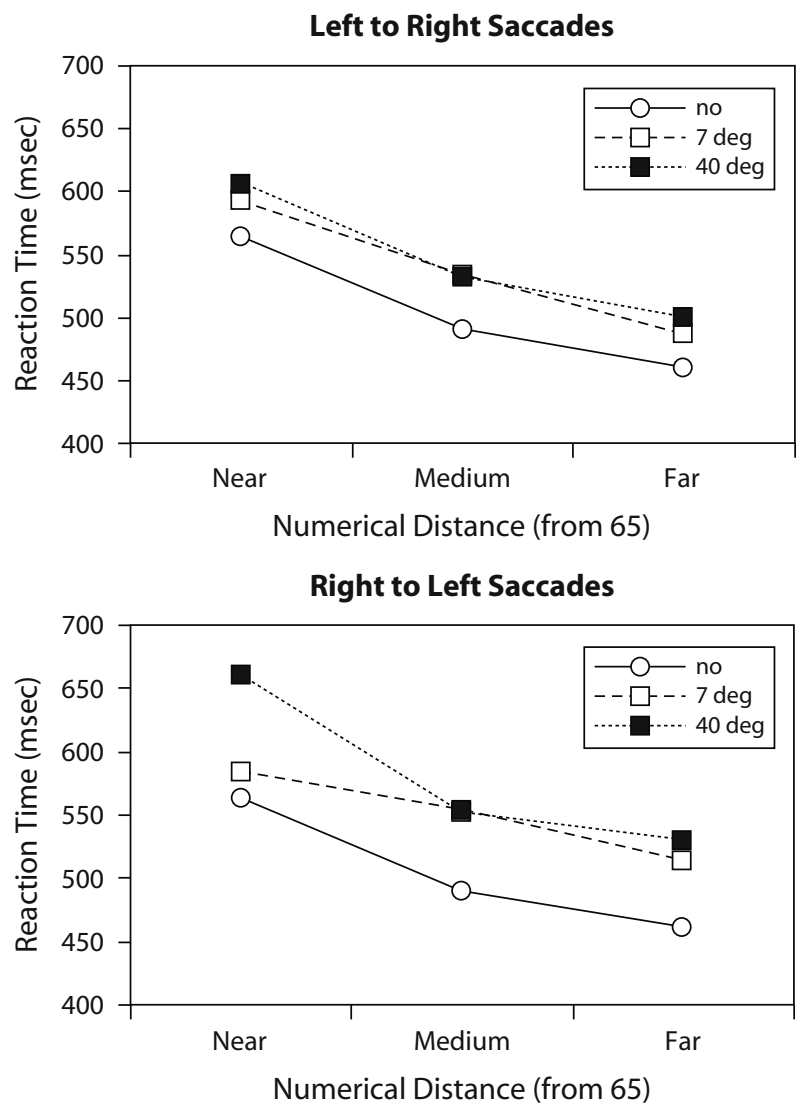

Figure 2. Reaction time (RT) in the magnitude comparison task as a function of saccade direction, saccade distance, and numerical distance for the $7^{\circ}$-saccade and $\mathbf{4 0}^{\circ}$-saccade conditions, along with RT for the no-saccade condition.

In other words, magnitude comparison is suppressed during saccades, but only for numbers close to the standard (numbers from 60 to 69) and only when the eyes move to the left. Given that the difference in duration between $7^{\circ}$ and $40^{\circ}$ saccades was $70 \mathrm{msec}$, the RT difference of 77 msec suggests that suppression was complete. ${ }^{1}$

The accuracy data (shown in Table 1) were consistent with the RT data. Accuracy increased with increasing numerical distance $\left[F(2,30)=5.9, M S_{\mathrm{e}}=0.014, p<.01\right]$, and this interacted with saccade direction $[F(2,30)=3.5$, $\left.M S_{\mathrm{e}}=0.05, p<.05\right]$; accuracy increased more as numerical distance increased when the eyes moved to the right ( $87.0 \%$ for near vs. $97.4 \%$ for far) rather than to the left $(92.1 \%$ for near vs. $95.8 \%$ for far). No interactions with saccade distance approached significance, however (all $F \mathrm{~s}<1.1$ ).

Parity judgments during saccades. As discussed in the introduction, the dual-task interference hypothesis predicts that saccades should not interfere with judging whether a number is odd or even, because this judgment relies on verbal processing and conceptual knowledge in the left frontal and temporal language areas rather than on spatial processing in the dorsal stream. Performance on the parity judgment task allows us to evaluate any impact that saccade distance and saccade direction might have 
Table 1

Percentage Correct in the Magnitude Comparison Task As a Function of Saccade Direction (Right vs. Left), Saccade Distance $\left(7^{\circ}\right.$ vs. $\left.4^{\circ}\right)$, and Numerical Distance (Near, Medium, Far)

\begin{tabular}{lcccc}
\hline $\begin{array}{c}\text { Saccade } \\
\text { Direction }\end{array}$ & $\begin{array}{c}\text { Saccade } \\
\text { Distance }\end{array}$ & Near & Medium & Far \\
\hline Right & $7^{\circ}$ & 88.1 & 90.3 & 97.4 \\
Right & $40^{\circ}$ & 85.9 & 94.7 & 97.4 \\
Left & $7^{\circ}$ & 93.6 & 94.0 & 94.9 \\
Left & $40^{\circ}$ & 90.6 & 92.1 & 96.7 \\
\hline
\end{tabular}

on number processing in general, however, divorced from magnitude comparison. For example, because our subjects were native English speakers who mostly move their eyes from left to right while reading, it is conceivable that saccade direction might affect all number processing tasks even when they do not involve magnitude comparison. So, to ensure that the interference we observed in the magnitude comparison task was due to magnitude comparison per se and not to other possible stimulus or response factors associated with numbers, we analyzed the data from the parity judgment task in the same way that we analyzed the magnitude comparison data, in terms of saccade direction (right vs. left), saccade distance $\left(7^{\circ}\right.$ vs. $\left.40^{\circ}\right)$, and numerical distance (near, medium, far).

Figure 3 shows the mean RT in the parity judgment task as a function of saccade direction, saccade distance, and numerical distance; the results of the no-saccade control condition are included for comparison. As in magnitude comparison, RT was longer in the dual-task condition (parity judgment during saccades) than in the single-task control condition (parity judgment without saccades); this difference presumably reflects additional executive processing time required in the dual-task condition in order to schedule and organize the operation of the two tasks and does not speak to the question of whether processing is suppressed during saccades per se. Suppression during saccades was evaluated in a three-way ANOVA with the factors saccade direction (right vs. left), saccade distance $\left(7^{\circ}\right.$ vs. $\left.40^{\circ}\right)$, and numerical distance (near, medium, far). No main effects or interactions were significant in this analysis (all $F_{\mathrm{S}}<2$ ). No main effects or interactions (all $F_{\mathrm{s}}<2$ ) were significant in a corresponding ANOVA conducted on the accuracy data, either (see Table 2).

In sum, as predicted, saccades had no effect on judging whether numbers were odd or even. This supports the hypothesis that saccades interfere only with dorsal-stream processes and also serves to demonstrate that the detrimental effect of saccades on magnitude comparison was due to magnitude comparison and not to some other potential interaction between saccades and number processing.

\section{DISCUSSION}

Previous research has suggested that saccadic eye movements interfere selectively with cognitive processes that rely on the dorsal stream. The results of the present research provide additional support for this conclusion by showing that magnitude comparison (a task that relies on the parietal cortex) is suppressed during saccades under certain circumstances, whereas parity judgment (a task that does not rely on the dorsal stream) is not suppressed at all. On each trial in the parity judgment task, subjects were presented with a two-digit number, and they indicated whether the number was odd or even. On dual-task trials, they made this decision while moving their eyes either $7^{\circ}$ or $40^{\circ}$ to the left or to the right. Saccade distance and saccade direction had no effect on performance; that is, subjects responded just as quickly and just as accurately regardless of how far they moved their eyes or in which direction. In contrast, in the magnitude comparison task, subjects indicated whether the two-digit number was greater than or less than 65 . Saccade distance had no effect on RT when the eyes moved from left to right, but RT for numbers close to 65 was $77 \mathrm{msec}$ slower on $40^{\circ}$-saccade trials than on $7^{\circ}$-saccade trials when the eyes moved from right to left. The difference in saccade duration between $7^{\circ}$ and $40^{\circ}$ saccades was $70 \mathrm{msec}$, so the RT difference of $77 \mathrm{msec}$ suggests that suppression was complete. Saccades made to the left during magnitude comparison also had a lower average velocity than did saccades made to the right (in comparison with a saccade-only control condition), suggesting not only that saccades interfered

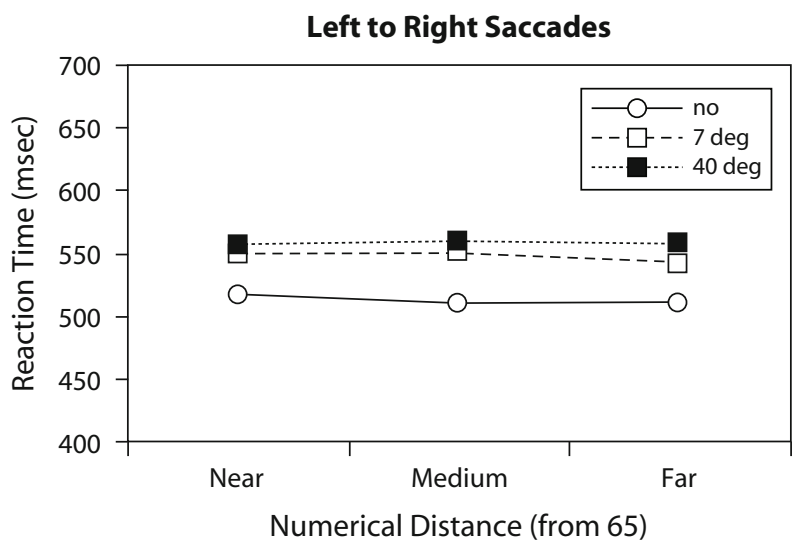

Right to Left Saccades

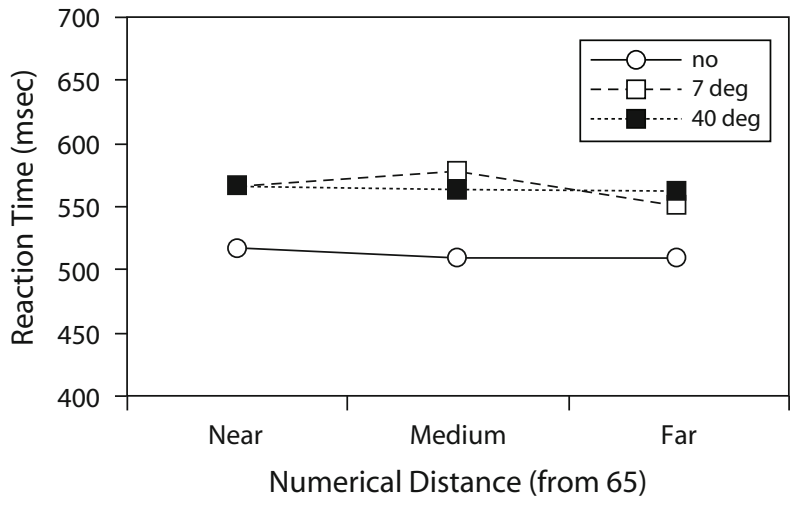

Figure 3. Reaction time (RT) in the parity judgment task as a function of saccade direction, saccade distance, and numerical distance for the $7^{\circ}$-saccade and $40^{\circ}$-saccade conditions, along with RT for the no-saccade condition. 
Table 2

Percentage Correct in the Parity Judgment Task As a Function of Saccade Direction (Right vs. Left), Saccade Distance ( $7^{\circ} \mathrm{vs}$. $4^{\circ}$ ), and Numerical Distance (Near, Medium, Far)

\begin{tabular}{lcccc}
\hline $\begin{array}{c}\text { Saccade } \\
\text { Direction }\end{array}$ & $\begin{array}{c}\text { Saccade } \\
\text { Distance }\end{array}$ & Near & Medium & Far \\
\hline Right & $7^{\circ}$ & 94.9 & 96.1 & 96.4 \\
Right & $40^{\circ}$ & 97.7 & 96.1 & 97.1 \\
Left & $7^{\circ}$ & 92.2 & 93.2 & 96.1 \\
Left & $40^{\circ}$ & 93.4 & 95.7 & 96.1 \\
\hline
\end{tabular}

with magnitude comparison, but also that magnitude comparison interfered with saccade execution as well. No such effect was found for parity judgments.

Why was interference in magnitude comparison found only for saccades to the left and only for numbers close to the standard? Several investigators have found that parietal activation in magnitude comparison is often larger in the right than in the left hemisphere (Chochon et al., 1999; Dehaene, 1996; Pinel et al., 2001), and the right parietal cortex is instrumental in programming saccades to the left (e.g., Behrmann, Ghiselli-Crippa, \& Dimatteo, 2001/2002; Colby \& Goldberg, 1999; Pierrot-Deseilligny, Rivaud, Gaymard, Muri, \& Vermersch, 1995); thus, there should be greater dual-task interference between magnitude comparison and saccades when saccades are made to the left as opposed to the right. Furthermore, the amount of parietal activation during magnitude comparison is highest for numbers close to the standard, and it diminishes as numerical distance increases (Pinel et al., 2001); so the greatest amount of dual-task interference between saccades and magnitude comparison should occur for numbers close to the standard. Thus, as is frequently the case in dual-task situations, perhaps saccades interfere with magnitude comparison only when the processing demands of the two tasks exceed some threshold.

The fact that interference with magnitude comparison was found only for saccades to the left raises the question of whether our results might have been due to processes associated with reading as opposed to magnitude comparison processes in the parietal cortex. That is, readers of English are accustomed to moving their eyes to the right while reading; because the stimulus number was read in our experiment, one might speculate that the interference we observed for saccades to the left was due to subjects' relative lack of experience with this situation. The results of the parity judgment condition rule out this hypothesis. The parity judgment task used exactly the same stimuli as were used in the magnitude comparison task, but saccades caused no interference with parity judgment. If the interference observed in the magnitude comparison task were due to some sort of reading bias, then interference should have been observed in the parity judgment task as well. The notion that saccades to the left might be inherently more difficult than saccades to the right is also disproved by several aspects of the eye movement data. For example, saccade direction had no effect on saccade latency, saccade distance, or average saccade velocity in the saccade-only control conditions. Furthermore, saccade direction had no effect on saccade latency in the dual-task conditions either (saccade latency was actually slightly faster to the left than to the right $-277 \mathrm{msec}$ vs. $287 \mathrm{msec}$ in the magnitude comparison task and $272 \mathrm{msec}$ vs. $279 \mathrm{msec}$ in the parity judgment task - but this difference was not significant in either case; $F<1.3$ ).

The fact that interference was found only for saccades to the left and only for numbers close to the standard (i.e., only for numbers from 60 to 69 ) raises the question of whether potential differences in fixation patterns or attentional allocation between left and right saccades (rather than magnitude comparison processes in the parietal cortex) might account for our results. That is, numbers from 60 to 69 are the only numbers in which both digits have to be compared to the standard (for the other numbers, the first, or tens, digit specifies whether the number is greater than or less than 65). One might be tempted to speculate that the interference we observed for numbers close to the standard when the eyes moved to the left was due to subjects' fixating in the "wrong" place or attending to the wrong digit location (i.e., the leftmost digit) when they were about to move their eyes to the left, and that a time-consuming shift of either the eyes or visual attention to the rightmost digit would be required on saccades to the left but not on saccades to the right (because the rightmost digit on saccades to the right is in line with the direction of the saccade). Several facts about eye movement behavior in the magnitude comparison task argue against this possible explanation for our results, however. First, there was no effect of numerical distance on fixation location; that is, the fixation location when the eyes moved from right to left was the same (within one screen pixel) regardless of whether the stimulus number was near, medium, or far with respect to the standard. Second, there was no effect of numerical distance on saccade latency; saccades to the left had a latency of $276 \mathrm{msec}$ for near stimuli, $280 \mathrm{msec}$ for medium stimuli, and $270 \mathrm{msec}$ for far stimuli. If subjects had to shift their visual attention within the number to identify the rightmost digit when a near stimulus was presented, then one would expect saccade latency to be lengthened as a result. It is also important to note that suppression during saccades was defined by the difference between two leftward saccade conditions (i.e., RTs for $40^{\circ}$-saccade trials vs. $7^{\circ}$-saccade trials), which would seem to take into account any general differences in fixation patterns or allocation of visual attention that might exist between left and right saccades. Finally, the results of the parity judgment task are also inconsistent with this alternative interpretation of our results; in parity judgment, the rightmost digit is always the most relevant digit to attend to (because it determines whether a number is odd or even), but saccade direction had no effect at all on performance in the parity judgment task.

In sum, we conclude that our results were not due to reading biases or to differences in where people were attending, but rather to interference between saccaderelated operations and the cognitive process of magnitude comparison. How might saccades interfere with magnitude comparison? On the basis of a large number 
of PET, fMRI, ERP, and patient studies, Dehaene et al. (2003) have proposed that three neural circuits are involved in number processing. One is located in the left angular gyrus and is associated with verbal processing of numbers. A second, located in the horizontal segment of the intraparietal sulcus, constitutes a nonverbal, core quantity system, analogous to a mental number line. The third is a posterior superior parietal system of spatial and nonspatial attention, used for attending to specific quantities on the mental number line; the closer two quantities are on the mental number line, the greater the attention that is needed to discriminate them. Significantly, this region of the posterior superior parietal cortex is not restricted to processing numbers but is active during a variety of visuospatial tasks, including eye movements, attention movements, hand movements, mental rotation, and spatial working memory (Corbetta, Kincade, Ollinger, McAvoy, \& Shulman, 2000; Culham \& Kanwisher, 2001; Simon, Mangin, Cohen, Le Bihan, \& Dehaene, 2002). Given this overlap, we propose that the interfering effect of saccades on magnitude comparison occurs within the posterior superior parietal system, hindering the inspection of the mental number line during magnitude comparison. That is, saccades do not interfere with the mental number line per se, but rather with the allocation of attention that is required in order to compare quantities on the number line.

In conclusion, our results provide further support for the hypothesis that saccades interfere with dorsal-stream processes. In particular, cognitive saccadic suppression appears to arise from dual-task interference; because saccade generation and saccade execution rely on the parietal cortex, cognitive processes that also rely on the parietal cortex cannot be executed at the same time.

\section{AUTHOR NOTE}

This research was supported by NSF Grant BCS 01-32292. The authors thank Gary Dell, Steve Franconeri, David Sheinberg, and two anonymous reviewers for their comments on the research, and Richard Carlson and Jordan Wojcik for assistance with data collection. Address correspondence to D. E. Irwin, Department of Psychology, University of Illinois, 603 E. Daniel St., Champaign, IL 61820 (e-mail: irwin@uiuc.edu)

\section{REFERENCES}

Behrmann, M., Ghiselli-Crippa, T., \& Dimatteo, I. (2001/2002). Impaired initiation but not execution of contralesional saccades in hemispatial neglect. Behavioral Neurology, 13, 39-60.

Brockmole, J. R., Carlson, L. A., \& IrWIN, D. E. (2002). Inhibition of attended processing during saccadic eye movements. Perception \& Psychophysics, 64, 867-881

Burr, D. C., Morrone, M. C., \& Ross, J. (1994). Selective suppression of the magnocellular visual pathway during saccadic eye movements. Nature, 371, 511-513.

Chochon, F., Cohen, L., van de Moortele, P. F., \& Dehaene, S. (1999). Differential contributions of the left and right inferior parietal lobules to number processing. Journal of Cognitive Neuroscience, 11, 617-630

Colby, C., \& Goldberg, M. (1999). Space and attention in parietal cortex. Annual Review of Neuroscience, 22, 319-349.

Corbetta, M., Kincade, J. M., Ollinger, J. M., McAvoy, M. P., \& Shulman, G. L. (2000). Voluntary orienting is dissociated from target detection in human posterior parietal cortex. Nature Neuroscience, $\mathbf{3}$, 292-297.
Culham, J. C., \& Kanwisher, N. G. (2001). Neuroimaging of cognitive functions in human parietal cortex. Current Opinion in Neurobiology, 11, 157-163.

DeHAENE, S. (1996). The organization of brain activations in number comparison: Event-related potentials and the additive-factors method. Journal of Cognitive Neuroscience, 8, 47-68.

Dehaene, S., \& Cohen, L. (1991). Two mental calculation systems: A case study of severe acalculia with preserved approximation. Neuropsychologia, 29, 1045-1074.

Dehaene, S., Dupoux, E., \& Mehler, J. (1990). Is numerical comparison digital? Analogical and symbolic effects in two-digit number comparison. Journal of Experimental Psychology: Human Perception \& Performance, 16, 626-641.

Dehaene, S., Piazza, M., Pinel, P., \& Cohen, L. (2003). Three parietal circuits for number processing. Cognitive Neuropsychology, 20, 487-506.

GoodAle, M. A., \& Milner, A. D. (1992). Separate visual pathways for perception and action. Trends in Neurosciences, 15, 20-25.

HENDERSON, J. M. (1992). Identifying objects across saccades: Effects of extrafoveal preview and flanker object context. Journal of Experimental Psychology: Learning, Memory, \& Cognition, 18, 521-530.

Henderson, J. M., Pollatsek, A., \& Rayner, K. (1987). Effects of foveal priming and extrafoveal preview on object identification. Journal of Experimental Psychology: Human Perception \& Performance, 13, 449-463.

Hubbard, E., Piazza, M., Pinel, P., \& Dehaene, S. (2005). Interactions between number and space in parietal cortex. Nature Reviews Neuroscience, 6, 435-448.

IRWIN, D. E. (1998). Lexical processing during saccadic eye movements. Cognitive Psychology, 36, 1-27.

IRWIN, D. E. (2003). Eye movements and visual cognitive suppression. In B. H. Ross (Series Ed.) \& D. Irwin (Vol. Ed.), The psychology of learning and motivation: Volume 42. Cognitive vision (pp. 265-293). San Diego: Academic Press.

IRWIN, D. E., \& BROCKMOLE, J. R. (2000). Mental rotation is suppressed during saccadic eye movements. Psychonomic Bulletin \& Review, 7, 654-661.

Irwin, D. E., \& BRockmole J. R. (2004). Suppressing where but not what: The effect of saccades on dorsal- and ventral-stream visual processing. Psychological Science, 15, 467-473.

Irwin, D. E., \& Carlson-Radvansky, L. A. (1996). Suppression of cognitive activity during saccadic eye movements. Psychological Science, 7, 83-88.

Irwin, D. E., Carlson-Radvansky, L. A., \& Andrews, R. V. (1995). Information processing during saccadic eye movements. Acta Psychologica, 90, 261-273.

Matin, E. (1974). Saccadic suppression: A review and an analysis. Psychological Bulletin, 81, 899-917.

Matin, E., SHAO, K., \& BofF, K. (1993). Saccadic overhead: Informationprocessing time with and without saccades. Perception \& Psychophysics, 53, 372-380.

Merigan, W. H., \& Maunsell, J. H. (1993). How parallel are the primate visual pathways? Annual Review of Neuroscience, 16 369-402.

Mishin, M., Ungerleider, L. G., \& Macko, K. A. (1983). Object vision and spatial vision: Two cortical pathways. Trends in Neurosciences, 6, 414-417.

Pierrot-Deseilligny, C., Rivaud, S., Gaymard, B., Muri, R., \& Vermersch, A. (1995). Cortical control of saccades. Annals of Neurology, 37, 557-567.

Pinel, P., Dehaene, S., Rivière, D., \& Le Bihan, D. (2001). Modulation of parietal activation by semantic distance in a number comparison task. NeuroImage, 14, 1013-1026.

RAYNER, K. (1978). Eye movements in reading and information processing. Psychological Bulletin, 85, 618-660.

RAYNER, K. (1998). Eye movements in reading and information processing: Twenty years of research. Psychological Bulletin, 124, 372-422.

Sanders, A. F., \& Houtmans, M. J. M. (1985). There is no central stimulus encoding during saccadic eye shifts: A case against general parallel processing notions. Acta Psychologica, 60, 323-338.

Sanders, A. F., \& Rath, A. M. (1991). Perceptual processing and speed-accuracy trade-off. Acta Psychologica, 77, 275-291. 
SCHall, J. D. (1995). Neural basis of saccade target selection. Reviews in the Neurosciences, 6, 63-85.

Simon, O., Mangin, J. F., Cohen, L., Le Bihan, D., \& Dehaene, S. (2002). Topographical layout of hand, eye, calculation, and languagerelated areas in the human parietal lobe. Neuron, 33, 475-487.

van Duren, L. (1993). Central stimulus processing during saccadic eye movements. In G. d'Ydewalle \& J. Van Rensbergen (Eds.), Perception and cognition: Advances in eye-movement research (pp. 23-35). Amsterdam: North-Holland.

van Duren, L., \& SANDERS, A. F. (1995). Signal processing during and across saccades. Acta Psychologica, 89, 121-147.

Volkmann, F. C. (1986). Human visual suppression. Vision Research, 26, 1401-1416.

WINER, B. J. (1971). Statistical principles in experimental design. New York: McGraw-Hill.

Zuber, B. L., \& STARK, L. (1966). Saccadic suppression: Elevation of visual threshold associated with saccadic eye movements. Experimental Neurology, 16, 65-79.

\section{NOTE}

1 . We were concerned that the difference in latency between $7^{\circ}$ (297$\mathrm{msec})$ and $40^{\circ}(268-\mathrm{msec})$ saccades might have contributed to the RT differences that were observed; because the eyes moved sooner on $40^{\circ}$ saccade trials, cognitive processing would have been interrupted at an earlier moment in time on these trials than on $7^{\circ}$-saccade trials. To examine this, we conducted a median split based on saccade latency for the $7^{\circ}$-saccade trials and reanalyzed the RT data using all of the $40^{\circ}$-saccade trials and only the $7^{\circ}$-saccade trials in which saccade latency was less than the median. The mean saccade latency on these $7^{\circ}$-saccade trials was $270 \mathrm{msec}$, very similar to the latency observed on $40^{\circ}$-saccade trials $(268 \mathrm{msec})$. An ANOVA of the RT data from these trials produced results entirely consistent with those reported in the main analysis; the three-way interaction of saccade direction, saccade distance, and numerical distance was significant in this analysis $\left[F(2,30)=4.8, M S_{\mathrm{e}}=\right.$ $3,404, p<.02]$. The error term from this interaction was used to construct a $95 \%$ confidence interval for the difference between two means; this value was $\pm 37.5 \mathrm{msec}$. Thus, as in the analysis based on all of the data, saccade distance had no significant effect on RT at any numerical distance when the eyes moved to the right; when the eyes moved to the left, however, RT for numbers close to the standard (i.e., near trials) was significantly slower when subjects made $40^{\circ}$ saccades $(660 \mathrm{msec})$ than when they made $7^{\circ}$ saccades $(560 \mathrm{msec})$.

(Manuscript received August 5, 2005; revision accepted for publication August 7, 2006.) 